\title{
Associative memory with spatiotemporal chaos control
}

\author{
Masanori Kushibe, Yun Liu, and Junji Ohtsubo \\ Faculty of Engineering, Shizuoka University, 3-5-1 Johoku, Hamamatsu, 432 Japan
}

(Received 18 December 1995)

\begin{abstract}
Control of spatiotemporal chaos in a neural network with discrete time and continuous state variables is investigated. The chaos control is performed with the knowledge of only a part of the target information in the memory patterns. The success rate for the pattern associations and the dependence of the search time on the sampling number in the proposed chaos neural network are studied. By the introduction of the reinforcement factor in the learning process, the recognition rate of the network can be much enhanced. Random and regular samplings of the pattern for the control are tested and the successful results of the associations are demonstrated. The chaotic behavior and recalling ability of the system are evaluated based on the analysis of the Lyapunov spectrum of the network.
\end{abstract}

PACS number(s): 05.45.+b, 05.50.+q, 87.10.+e

\section{INTRODUCTION}

Recent research in physiological science fields revealed the existence of chaotic dynamics in some biological neural systems [1,2], and chaos seems to be essential in such systems. Even in high life forms, such as in the operations of the neurons in the human brain, it is recognized that there exists a certain chaotic dynamics in the networks. The question naturally arises whether such chaotic dynamics plays a functional role in the information processing of neural networks. At the same time, from a theoretical viewpoint of physics, nonlinear dynamics and spatiotemporal chaos have been investigated in a variety of extended systems, including asymmetric neuron models [3-9]. For example, a chaotic neural network model that responds chaotically itself has been proposed by Aihara, Tanabe, and Toyoda [6] by extending the Nagumo-Sato model [7]. As an application of chaotic dynamics for the information processing of neural networks, Toyoda et al. [8] proposed an associative model with a chaotic neural network and indicated that such a system can be consisted of an autonomous memory model and the model can associate memory patterns nonperiodically. Nakamura and Nakagawa [9] realized an associative memory that can avoid the problems of local minima by the parameter control technique with a chaotic neural network.

In this paper, we analyze the characteristic features of the spatiotemporal chaos in a chaotic neural network and apply it to an associative memory. The method proposed by Nakamura and Nakagawa [9] required the information for the complete memory pattern to associate the target pattern. Here, we propose an associative memory network with chaos control by using only a part of the target information. It is found that the effect of the relative inhibitory constant in the previous network is too strong to recall the correct pattern in our model. Therefore, we add the reinforcement factor in the learning process to enhance the firing rate of neurons when their output state approaches the neighborhood of the target

\footnotetext{
${ }^{*}$ Also at Graduate School of Electronic Science and Technology, Shizuoka University, 3-5-1 Johoku, Hamamatsu, 432 Japan. FAX: +81-53-478-1251. Electronic address: ohtsubo@eng.shizuoka.ac.jp
}

pattern. By the introduction of this term, the recognition rate of the network can be much enhanced. The dependences of the success rate and the search time on the sampling number in the proposed system are extensively studied. The relevance between the chaotic dynamics and recalling capability of the system are investigated based on the analysis of the Lyapunov exponents of the network. Successful associations are obtained for both the random and regular sampling techniques used in the spatiotemporal chaos control. It is noted that the recovery of a pattern from the knowledge of the partial information is very important for the practical applications of neural networks, such as pattern compressions and coded data communications.

\section{CHAOTIC NEURAL NETWORK}

For spatiotemporal chaos control, we employ a chaotic neural network model proposed by Aihara et al. To begin with, let us briefly describe the model for later use. The model is defined by the equation with discrete time and continuous state as follows:

$$
\begin{gathered}
y_{i}(n+1)=k y_{i}(n)+\sum_{j=1}^{M} w_{i j} x_{j}(n)-\alpha x_{i}(n)+a_{i}, \\
x_{i}(n+1)=f\left(y_{i}(n+1)\right),
\end{gathered}
$$

where $y_{i}(n)$ and $x_{i}(n)$ are the internal state and the output of the $i$ th neuron, respectively, $M$ is the total number of neurons in the network, $k$ is a memory constant with $0 \leqslant k<1$ to guarantee the convergence of the iteration, $\alpha$ is the relative inhibitory constant $(\alpha>0)$ to restrain a firing after excitations of the neurons, $w_{i j}$ is the connection matrix component, and $a_{i}$ is the external input of the $i$ th neuron. The function $f(y)$ is a threshold function and usually takes the sigmoid form as

$$
f(y)=\tanh \left(\frac{y}{\varepsilon}\right),
$$

where $\varepsilon$ is a certain parameter called the temperature of neurons. The second term of the right-hand side of Eq. (1) is the 
ordinary Hopfield term, and the model defined by Eqs. (1)(3) returns in the Hopfield model for the special case of $k=\alpha=a_{i}=0$ [10]. The last term is a bias to the neuron and the rest are introduced to generate chaotic dynamics in the system. Therefore, it is considered that the chaotic neural network model is introducing the chaotic dynamics into the Hopfield model as a natural expansion of the latter one.

The chaotic characteristics of the network can be measured by the Lyapunov spectrum [11]. Here, we generalize Eq. (1) as a mapping,

$$
\mathbf{y}(n+1)=\mathbf{G}\{y(n)\},
$$

where $\mathbf{G}$ is the nonlinear mapping and $\mathbf{y}(n)$ $=\left(y_{1}, y_{2}, \ldots, y_{M}\right)$. When $\mathbf{G}$ is known, we iterate the following equation starting from an orthogonal vector $\mathbf{u}(n)=\left(u_{1}, u_{2}, \ldots, u_{M}\right)$

$$
\boldsymbol{\nu}(n+1)=\mathbf{D G}\{\mathbf{y}(n)\} \mathbf{u}(n),
$$

where DG is the Jacobian matrix of the mapping $\mathbf{G}$. To calculate Lyapunov spectrum, $\boldsymbol{\nu}(n+1)$ is orthogonalized by the Gram-Schmit method and renormalized as follows;

$$
\nu_{i}^{\prime}(n+1)=\nu_{i}(n+1)-\sum_{j=1}^{i-1}\left\langle\nu_{i}(n+1), u_{j}(n+1)\right\rangle u_{j}(n+1),
$$

$$
u_{i}(n+1)=\frac{\nu_{i}^{\prime}(n+1)}{\left|\nu_{i}^{\prime}(n+1)\right|}
$$

where \langle\rangle denotes an inner product. Then, the Lyapunov exponent $\lambda_{i}$ is defined as

$$
\lambda_{i}=\lim _{K \rightarrow \infty} \frac{1}{K} \sum_{n=1}^{K} \log \left|\nu_{i}^{\prime}(n+1)\right| .
$$

If, at least, the maximum Lyapunov exponent has a positive value, one can confirm that the network is in chaos [11].

\section{CHAOS CONTROL FOR ASSOCIATIVE MEMORY}

Let us consider the chaotic dynamics of the present network model for the associative memory. First, the connection matrix $w_{i j}$ is defined by the autocorrelation matrix as

$$
w_{i j}=\frac{1}{L} \sum_{r=1}^{L} e_{i}^{(r)} e_{j}^{\dagger(r)}
$$

where $L$ is the number of embedded patterns. $\mathbf{e}^{(r)}$ and $\mathbf{e}^{\dagger(r)}$ are vectors of the embedded pattern and its adjoint, respectively, and they satisfy the following condition:

$$
\sum_{i=1}^{M} e_{i}^{(r)} e_{i}^{\dagger(s)}=\delta_{r s}
$$

where $\delta$ is the Kronecker delta function. The embedded patterns of $L=6$ with the number of neurons of $M=100$ used in the simulations are shown in Fig. 1. To investigate the chaotic dynamics of the network, a bifurcation diagram of the maximum Lyapunov exponent versus the memory constant

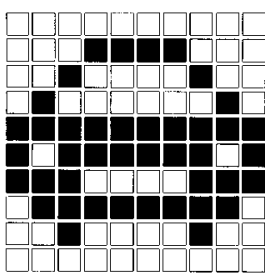

CAR

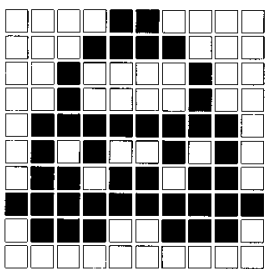

UFO

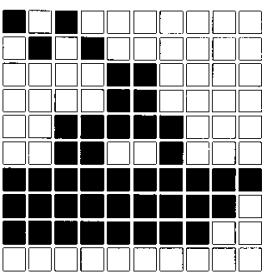

SHIP

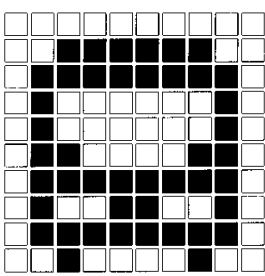

BUS

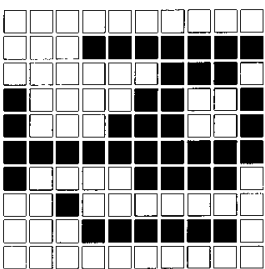

HELICOPTER

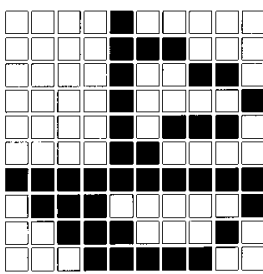

YACHT
FIG. 1. Set of patterns embedded in the network.

$k$ and the relative inhibitory constant $\alpha$ is shown in Fig. 2. The black area in the figure corresponds to the positive values of the maximum Lyapunov exponent, $\lambda_{\max }>0$, whereas the white area is for the negative values. The results show that the network dynamics is dependent on the network parameters $k$ and $\alpha$. Similar plots of the bifurcations are obtained for different values of $a_{i}$, although these are not plotted here.

Next, we consider the spatiotemporal control of the chaotic behaviors in the present network and apply it to the

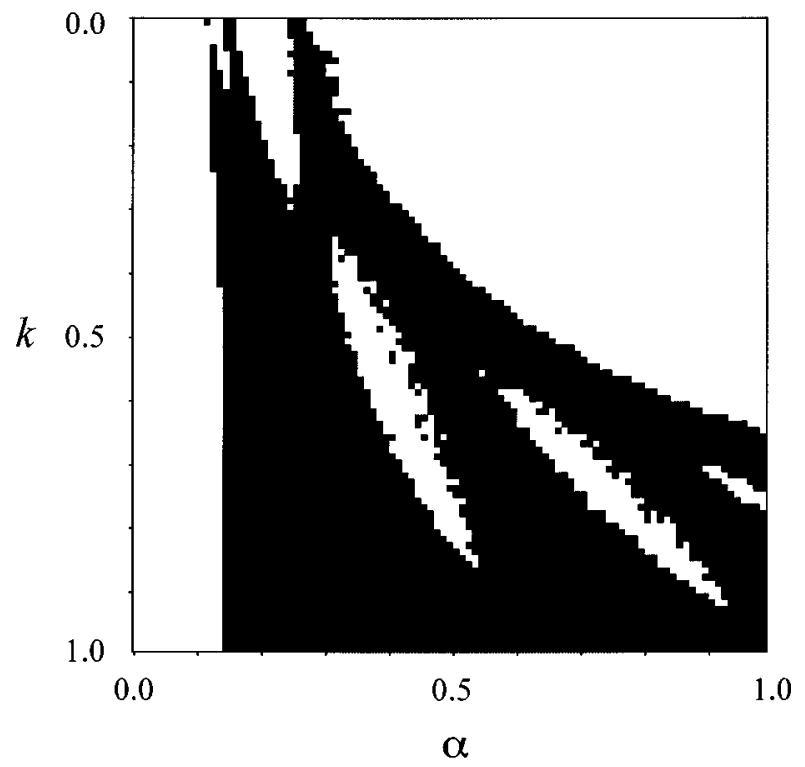

FIG. 2. Lyapunov exponent diagram versus memory constant $k$ and relative inhibitory constant $\alpha . M=100, \varepsilon=0.02$, and $a_{i}=0.2$ (for all $i$ ). 
associative memory by controlling the network parameter values. In the control configuration, the values of the network parameters are varied as a function of a control signal $\mu^{(\tau)}(n)[9]$,

$$
\begin{gathered}
k(n)=\left[1-\mu^{(\tau)}(n)\right] k(0), \\
\alpha(n)=\left[1-\mu^{(\tau)}(n)\right] \alpha(0), \\
a_{i}(n)=\left[1-\mu^{(\tau)}(n)\right] a_{i}(0),
\end{gathered}
$$

where $k(0), \alpha(0)$, and $a_{i}(0)$ are initial values of the control parameters and $\tau$ represents the suffix for one of the embedded patterns, i.e., the target pattern. There are a number of control methods to recall the correct memory pattern. Here we employ the method where the control signal is generated with an overlap between the output pattern and a randomly chosen part of the target pattern. The control signal is defined by

$$
\begin{gathered}
\mu^{(\tau)}(n)=\frac{1}{N} \sum_{i=1}^{N} g^{(\tau)}\left(n, R_{i}\right), \\
g^{(\tau)}(n, i)=\operatorname{sgn}\left[x_{i}(n) e_{i}^{(\tau)}\right] \exp \left\{\left|\frac{x_{i}(n)}{e_{i}^{(\tau)}}\right|-1\right\},
\end{gathered}
$$

where $N(<M)$ is the sampling number and $R_{i}$ is an integer number distributed randomly within 1 to $M$. The function $g^{(\tau)}(n, i)$ is defined in a way that it outputs +1 for the correct recollection and -1 for the completely inverse recollection. It is found from Fig. 2 that the network changes from chaos to stable states when the control signal $\mu^{(\tau)}(n)$ approaches unity. In other words, the system changes from the chaotic neural network model to the Hopfield model when $k, \alpha$, and $a_{i}$ are close to zero. Therefore, the network defined here has both the possibility to escape from spurious states with chaotic transition and the function as a Hopfield model to associate the target pattern when the output of the network is close to the target pattern.

The value of the parameter $\alpha$ that restrains the excitation after the firing of a neuron exhibits a crucial influence on the performance of the present neural network model. To avoid successfully spurious states in the recalling process, the value of $\alpha$ must be fairly large. Meanwhile, for a large value of $\alpha$ and a small sampling number, the system sometimes fails to get out from the chaotic states and cannot reach the Hopfield network. So a learning algorithm [5] is employed to improve the memory search ability of the network. This algorithm adapts the external input value $a_{i}$ during the search for the target pattern as

$$
\begin{gathered}
\text { if } \quad \mu^{(\tau)}(n) \geqslant \gamma, \\
\text { then } \quad a_{i}=\left[1-\mu^{(\tau)}(n)\right]\left[a_{i}(0)+\Delta e_{i}^{(r)}\right] \quad(i \in T),
\end{gathered}
$$

where $\gamma$ is a certain positive threshold value between 0 and $1, \Delta$ is the reinforcement constant per learning process, and $T$ is a set of sampling neurons. With Eq. (16), Eq. (1) can be rewritten as

$$
\begin{gathered}
y_{i}(n+1)=k y_{i}(n)+\sum_{j=1}^{M} w_{i j} x_{j}(n) \\
-\left[\alpha x_{i}(n)-\Delta(n) e_{i}^{(r)}\right]+a_{i} \quad(i \in T), \\
y_{i}(n+1)=k y_{i}(n)+\sum_{j=1}^{M} w_{i j} x_{j}(n) \\
-\alpha x_{i}(n)+a_{i} \quad(i \notin T),
\end{gathered}
$$

where $\Delta(n)$ is defined as $\Delta(n)=\Delta\left[1-\mu^{(\tau)}(n)\right]$. Thus, the effect of this learning algorithm facilitates the firing probability of neurons whose output state is close to the target $\left[\mu^{(\tau)}(n) \geqslant \gamma\right]$ and makes the retrieval of the target pattern more easily and rapidly. Thus, the network easily gets out from the chaotic dynamics in the neighborhood of the target pattern. On the other hand, the introduction of this term has no influence on the network dynamics when the output state is far form the target pattern. In the next section, some numerical results for the memory search are demonstrated by the proposed chaos control.

\section{SIMULATION RESULTS AND DISCUSSIONS}

The numerical simulations have been performed by using the proposed network model for $M=100$. Six images ( $L=6$, Fig. 1) have been embedded in the network. The black and white points in the figure correspond to the states with $e_{i}^{(r)}(n)=+1$ and -1 , respectively. The initial values of the parameters, $k(0), \alpha(0)$, and $a_{i}(0)$, are set to appropriate values for which chaos is observed in the network. In the simulations, we put these parameters values into $0.9,0.6$, and 0.2 , respectively. The corresponding maximum Lyapunov exponent is evaluated as $0.3911(>0)$.

Figure 3 shows an example of the associative processes for the target pattern "yacht." In this case, the sampling density $N / M$ was chosen to be $\frac{1}{2}$ and the initial input was a random pattern. The reinforcement constant $\Delta$ was zero in this case. In the figure, the size of the square represents the proportion to the value of $\left|x_{i}(n)\right|$, where the black squares are for $x_{i}(n)>0$, while the white ones are for $x_{i}(n)<0$. Even starting from a random initial pattern, the memory search succeeded without trapping in local minima. Therefore, it is demonstrated that the present chaotic neural network can associate the target pattern by the appropriate chaos control.

Next, we investigate the success rate and search time for the sampling number of the present neural network model. The simulations of the memory search by the control were carried out for $n=0 \sim T_{\max }(=200)$. The success rate $S_{r}$ was defined by the ratio of the number of the successful trials to the total number of trials that are performed under different initial patterns. The average search time $S_{t}$ is defined by [5]

$$
S_{t}=\frac{\sum_{u=0}^{S} t_{u}}{S_{r} S}
$$

where $t_{u}$ is the time step required for the complete association of the target pattern and $S$ the total trial number. The results are shown in Fig. 4. For the elements of the known 

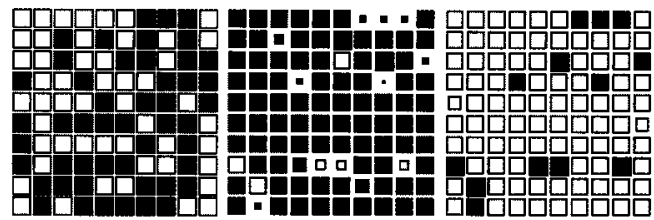

TIME $=0$

TIME=1

TIME $=2$

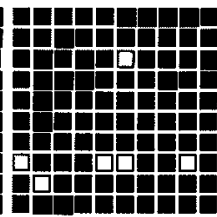

TIME $=7$
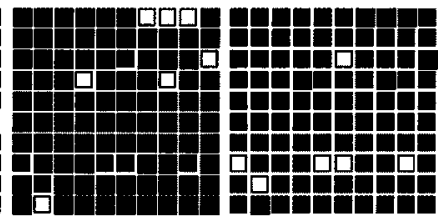

TIME $=3$

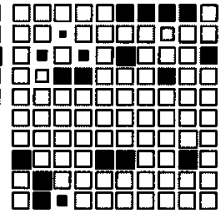

TIME $=8$
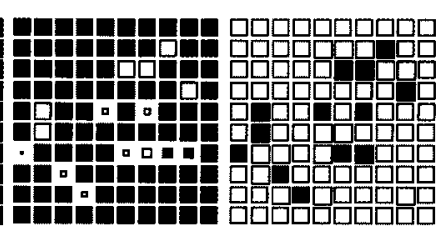

TIME $=107$

TIME $=108$
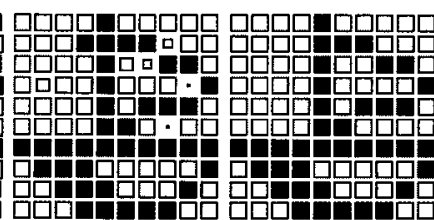

TIME $=113$

TIME $=112$

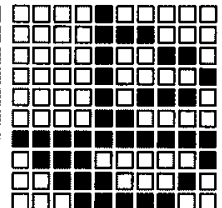

TIME $=117$

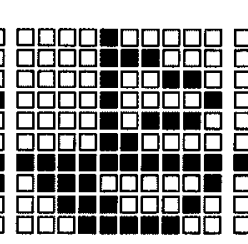

TIME $=118$

TIME $=118$
TIME $=4$

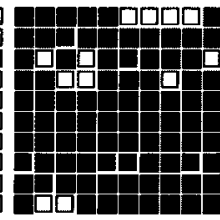

TIME $=9$

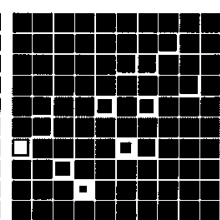

TIME $=109$

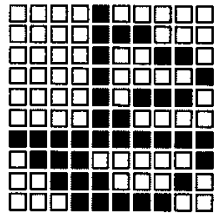

TIME $=115$
TIME=116

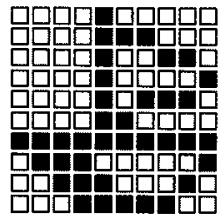

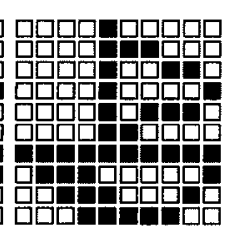

TIME $=114$
FIG. 3. Example of the associative process for the target pattern of "yacht." sampling neurons, we used the values which are exactly the same as the target pattern $e_{i}^{(r)}$ as initial states. Otherwise we used random and bipolar initial values as initial states. The target pattern was "car" in Fig. 1. The reinforcement constant $\Delta$ was varied as a parameter and four different values, $0.0,0.1,0.5$, and 0.9 , were used for the constant in the simulation. For comparison, the success rate for the Hopfield model was also calculated and displayed as black dots. In the absence of the reinforcement constant, the success rate is always small and the search time is long compared with other parameter values and the Hopfield model. However, with an appropriate choice of the reinforcement constant, e.g., $\Delta=0.5$, both the success rate and the search time are optimized and the correct recollection can be realized even for a small sampling number. For the Hopfield model, we generate the initial pattern where the number of neurons that is consistent with the target pattern is equal to the sampling number. For a sampling number above $50 \%$ of the total neurons, the successful recollection is always attained for all parameter conditions including the Hopfield model. The average search time is shown in Fig. 4(b). Here again the cha- otic neural network surpasses the Hopfield model for an appropriate value of $\Delta$. Especially for a small sampling number, the search time of the chaotic neural network is dramatically decreased compared with the Hopfield model.

Figure 5 shows the success rate for different $T_{\text {max }} s$ in the present neural network for $\Delta=0.0$ to test the dependence of the length for the search time. For the increase of the search time $T_{\text {max }}$, slight increase of the success rate can be seen in low sampling number, but the difference is small elsewhere. Obviously, this network does not improve the success rate as compared with the Hopfield model. One of the possible reasons why the recalling ability of the network with $\Delta=0.0$ is inferior to that of the Hopfield model is that the relative inhibitory factor $\alpha$ is too strong in the neural network dynamics. Due to this effect, the network state keeps on behaving chaotically and seldom visits the neighborhood of the target pattern. Therefore, without or with a small value of the reinforcement constant, the present network can avoid the trap in local minima, but it usually behaves chaotically or periodically without associating the target pattern.

Figure 6 shows the dependence of the success rate on the 


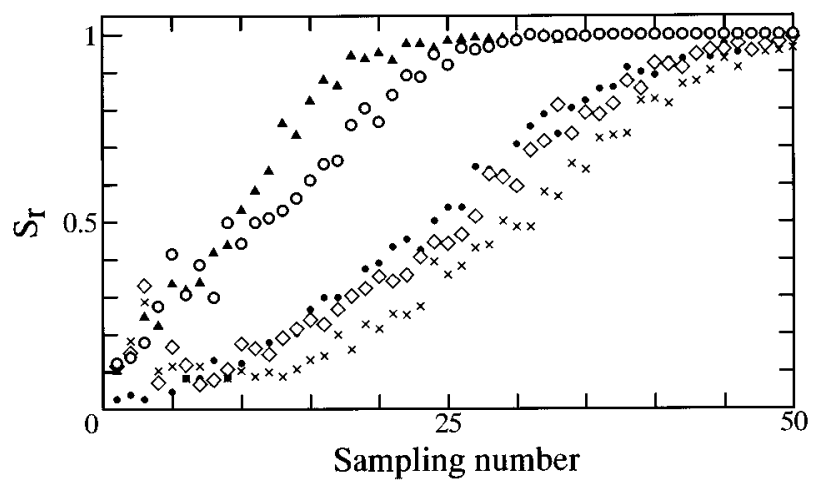

(a)

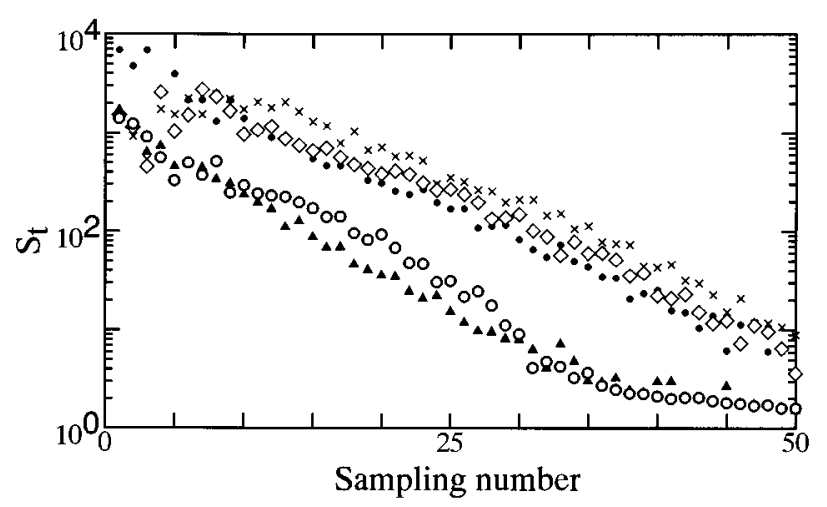

(b)

FIG. 4. Success rate $S_{r}$ (a) and search time $S_{t}$ (b) versus sampling number. 0 : Hopfield model, $\times: \Delta=0.0, \diamond: \Delta=0.1$, $\boldsymbol{\Delta}: \Delta=0.5$, and $\bigcirc: \Delta=0.9$.

reinforcement constant for sampling numbers of $N=50,20$, and 5. When the sampling number is over 50, the proposed (open circles) model can successfully recall the correct patterns for all reinforcement constants and the success rate is compatible with that of the Hopfield model (upper straight broken line). For a moderate sampling rate $N=20$, the success rate greatly changes depending on the value of the re-

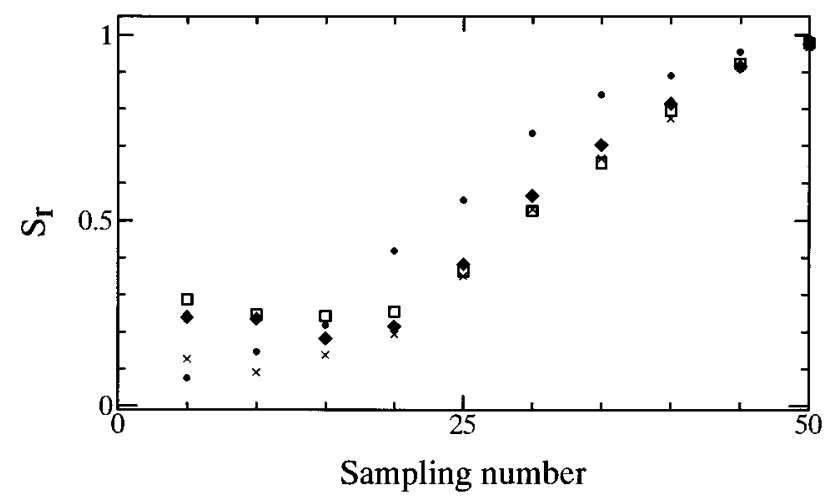

FIG. 5. Dependence of the success rate $S_{r}$ on the search step $T_{\max }$ with $\Delta=0.0$. O: Hopfield model, $\times: T_{\max }=200$,

$T_{\max }=5000$, and $\square: T_{\max }=10000$.

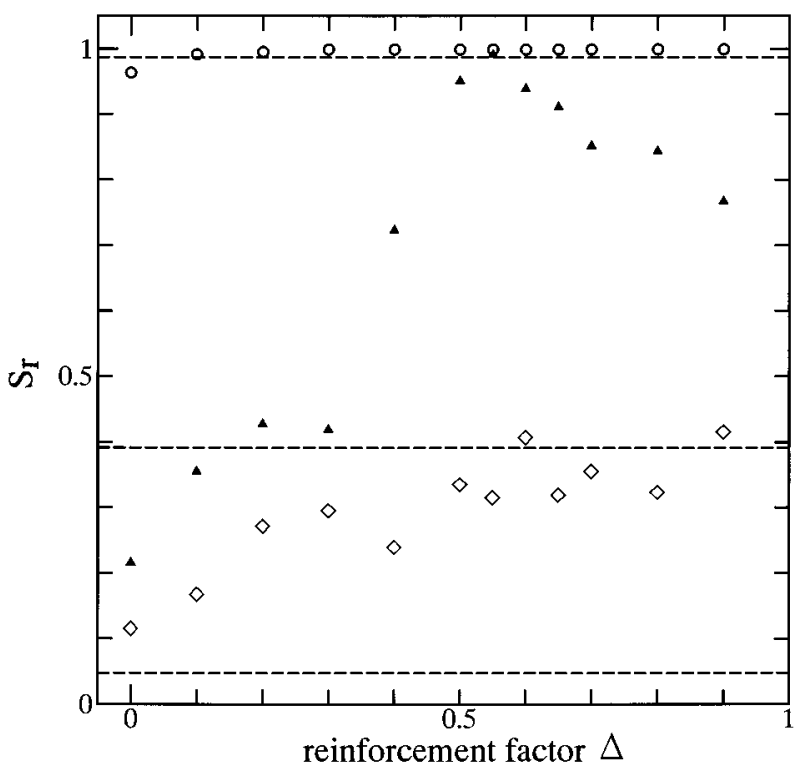

FIG. 6. Dependence of the success rate $S_{r}$ on the reinforcement factor. $\bigcirc: N=50, \boldsymbol{\Delta}: N=20$, and $\diamond: N=5$. Broken lines are the values for the Hopfield model for $N=50$ (upper line), $N=20$ (center line), and $N=5$ (lower line).

inforcement factor. The success rate for the Hopfield model is about 0.4 (middle straight broken line), while the maximum success rate of unity is attained in the present chaotic neural network model (solid triangles) at $\Delta=0.55$. Even for a small sampling number of $N=5$, the success rate of 0.4 is obtained in the chaotic neural network (open diamonds), while that of the Hopfield model (lower straight broken line) is merely 0.05 . The above results indicate that the association rate in the chaotic neural network is much improved by the appropriate choice of the reinforcement constant especially for moderate or small sampling numbers.

Finally, the effects of the chaotic dynamics on the association in the present network are investigated by comparing the Lyapunov exponents with the success rate. The bifurcation diagram of the Lyapunov exponents versus the system parameters $k(0)$ and $\alpha(0)$ have already been displayed in Fig. 2. Here we employ the same parameter conditions used there. Figure 7(a) shows the success rate versus the parameter value of $k(0)$ for the relative inhibitory constant of $\alpha(0)=0.6$ and the bias of $a_{i}(0)=0.2$ (for all $i$ ), and Fig. 7 (b) shows the Lyapunov exponents versus $k(0)$ that correspond to the variations for the cross section of the map at $\alpha(0)=0.6$ in Fig. 2. The sampling number is set to be $N=25$ and the value of the reinforcement factor is $\Delta=0.5$. The straight broken line in Fig. 7(a) denotes the success rate for the Hopfield model. One easily finds that the high success rate of recalling is obtained for the positive Lyapunov exponent region.

Figure 8 shows the effects of the inhibitory constant $\alpha$ on the success rate. Figure 8 (a) represents the plot of the success rate versus the inhibitory constant for the value of the memory constant of $k(0)=0.9$, and Fig. $8(\mathrm{~b})$ is the corresponding Lyapunov spectrum. The straight broken line again denotes the success rate for the Hopfield model. Although a high success rate is obtained for a positive Lyapunov expo- 


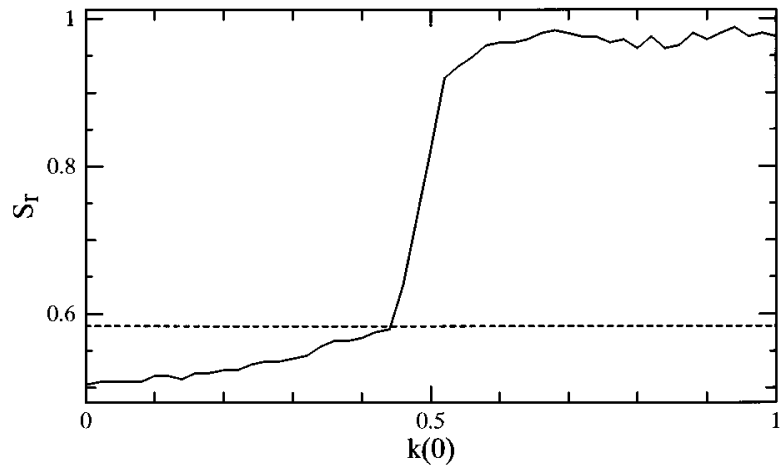

(a)

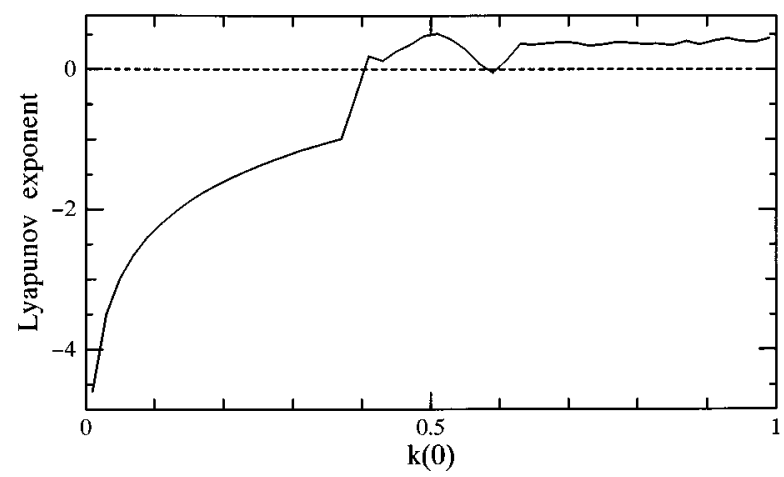

(b)

FIG. 7. Dependence of the success rate $S_{r}$ (a) and the Lyapunov exponent (b) on the initial memory constant $k(0) . \alpha(0)=0.6$ and $a_{i}(0)=0.2($ for all $i)$.

nent region $(\alpha=0.5-0.7)$, the positive Lyapunov exponents do not necessarily assure the high success rate of the association. The reason is considered as follows. Strictly speaking, the retrieval process depends on the variations of the control parameters in the recalling process, which change from the initially assigned values to zero when the network successfully associates the target pattern. So only the starting dynamics is not enough to explain the success rate of the network. To gain further insight into the association mechanism, we are now investigating the relation among the success rate, the retrieval process, and the variation of the control parameters, and the results will be presented in the future.

We also tried chaos control in the present neural network with regular sampling algorithm instead of random sampling. In this case, Eq. (14) is replaced by the following regular sampling function:

$$
\mu^{(\tau)}=\frac{1}{N} \sum_{i=1}^{M}\left\{\sum_{k=1}^{N} \delta(i-P k)\left[g^{(\tau)}(n, i)\right]\right\},
$$

where $P$ is the spatial sampling density. For the regular sampling algorithm, similar results of the associations and dependences of the success rate and the search time on the sampling number to those of the random sampling were obtained by using the same set of the memory patterns.

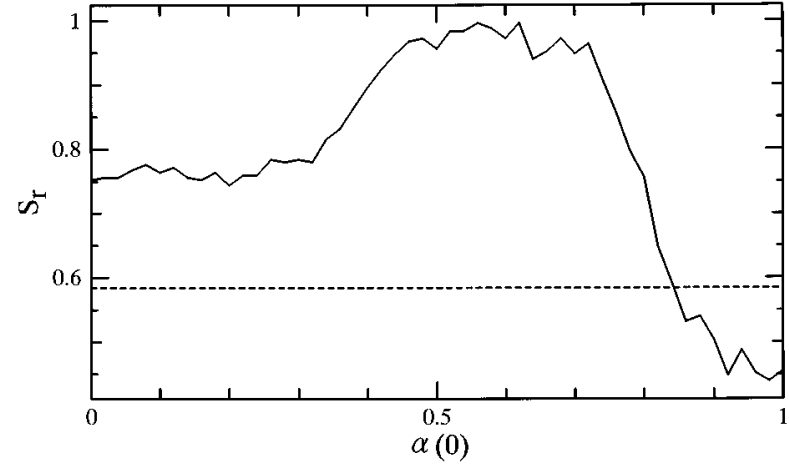

(a)

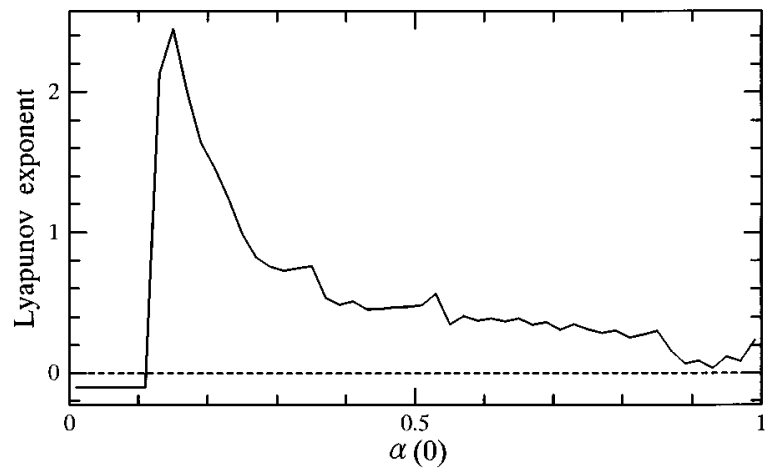

(b)

FIG. 8. Dependence of the success rate $S_{r}$ (a) and the Lyapunov exponent (b) on the initial relative inhibitory constant $\alpha(0)$. $k(0)=0.9$, and $a_{i}(0)=0.2$ (for all $i$ ).

\section{CONCLUSION}

We have applied the spatiotemporal chaos control to an associative memory search in a chaotic neural network. It has been shown that the association in the network with a partial knowledge of the target information is successfully performed with the parameter control of spatiotemporal chaos. By the proposed learning algorithm, the performance of the memory search has been greatly improved in comparison to the Hopfield model. The obtained results imply the possibility of avoiding the problems of local minima in neural networks by appropriately applying the flexible chaotic dynamics. In practice, it has been shown that the successful association can be achieved at least for a partial input of $20 \%$ of the total number of neurons. One of the possible applications for the proposed chaotic neural network and the method of the associative memory recalling is the data compression in the field of data communications.

The chaotic neural network used for the simulations may not be the best network for such a memory search problem. As a future issue, various chaotic neural networks should be tested for the better memory search ability and memory capacity. We have not mentioned the memory capacity of the present chaotic neural network in this paper. The chaotic neural networks can store more memory patterns than the conventional Hopfield network. Therefore, it seems worthwhile to investigate the memory capacity for such chaotic neural networks. 
[1] A. Babloyanz, J. M. Salazar, and C. Nicolis, Phys. Lett. 111A, 152 (1985).

[2] F. Gruneis, M. Nakao, M. Yamamoto, and H. Nakahama, Biol. Cybern. 60, 161 (1989).

[3] G. Hu and Z. Qu, Phys. Rev. Lett. 72, 68 (1994).

[4] T. Fukai and M. Shiino, Phys. Rev. Lett. 64, 1465 (1990).

[5] S. Nara, P. Davis, and H. Totsuji, Neural Networks 6, 963 (1993).

[6] K. Aihara, T. Tanabe, and M. Toyoda, Phys. Lett. A114, 333
(1990).

[7] J. Nagumo and S. Sato, Cybernetics 10, 155 (1972).

[8] M. Toyoda, M. Adachi, K. Shimizu, and K. Aihara, IEICE Technical Report NC89-8 (1989).

[9] K. Nakamura and M. Nakagawa, J. Phys. Soc. Jpn. 62, 2942 (1993).

[10] J. J. Hopfield, Proc. Natl. Acad. Sci. USA 79, 2254 (1982).

[11] T. Ikeguchi, K. Aihara, S. Itoh, and T. Utsunomiya, IEICE Trans. Fundamentals J78-A, 486 (1990). 